3. Davis GR (1993) Neoplasms of the stomach. In: Sleisenger MH, Fordtran JS (eds) Gastrointestinal diseases, 5 th edn. Saunders, Philadelphia, pp 763-789

4. Delbrück H, Severin M (1991) Auswirkungen der partiellen und totalen Gastrektomie auf das Gewichtsverhalten und die Postgastrektomie-Beschwerden. In: Delbrück H (Hrsg) Krebsnachsorge und Rehabilitation. Band 3: Magenkarzinom. Zuckschwerdt, München Bern Wien San Francisco, S 62-70

5. Deutsche Gesellschaft für Ernährung e.V. (1996) Ernährungsbericht. Henrich, Frankfurt

6. Deutsche Gesellschaft für Ernährung e.V. (2000) Ernährungsbericht. Henrich, Frankfurt

7. Enck P, Bielefeldt K, Rathmann W, Purrmann J, Tschöpe D, Erckenbrecht JF (1991) Epidemiology of faecal incontinence in selected patient groups. Int J Colorect Dis 6: 143-146

8. Faivre J, Benhamiche AM, Dancourt V (2002) Endoscopic monitoring after polypectomy. What efficacy? What rhythm? Presse Med 31: 128-131

9. Fennerty MB (2001) Barrett's esophagus. In: Irvine EJ, Hunt RH (eds) Evidence-based gastroenterology. Decker, Hamilton London, pp 40-50

10. Fremann D, Wolfram $G$ (2001) Längeres Leben mit Lebensqualität. Akt Ernaehr Med 26: 183-190

11. Gabriel SE, Jaakimainen L, Bombardier C (1991) Risk for serious gastrointestinal complications related to use of nonsteroidal anti-inflammatory drugs. A meta-analysis. Ann Intern Med 115: 787-796

12. Hansen JM, Hallas J, Lauritsen JM, Bytzer $P$ (1996) Nonsteroidal anti-inflammatory drugs and ulcer complication: a risk factor analysis for clinical decision-making. Scand J Gastroenterol 31: 126-130

13. Hansen JM, Bytzer P (2001) Prevention of NSAID induced gastroduodenal ulcers. Ugeskr Laeger 163: 6103-6105

14. Herrmann M (2001) Antibiotika-assoziierte Diarrhoe - die unterschätzte Krankheit. Verdauungskrankheiten 19: 220-233

15. Hotz J (1992) Tumoren des Magens. In: H. Goebell (Hrsg) Gastroenterologie, Teil C. Urban \& Schwarzenberg, München Wien Baltimore, S 454-465

16. Jostarndt L (1986) Die anale Kontinenz und ihre Störung. Die Gastroenterologische Reihe, Band 24. Kali-Chemie, Hannover, S 29

17. Kjellin A, Ramel S, Rossner S, Thor K (1996) Gastroesophageal reflux in obese patients is not reduced by weight reduction. Scand J Gastronterol 31: 1047-51
18. Lührmann P,Herbert $B$, Neuhäuser-Berthold $M$ (2001): Underreporting of energy intake in an elderly German population. Nutrition 17: 912-916

19. McFarland LV, Surawicz CM, Greenberg RN, Fekety R (1995) Prevention of beta-lactamassociated diarrhea by Saccharomyces boulardii compared with placebo. Am J Gastroenterol 90: 439-448

20. Painter NS, Burkitt DP (1975) Diverticular disease of the colon, a 20th century problem. Clin Gastroenterol 4: 3-21

21. Pollmann H, Hübner P, Steiner E, Zillessen E (1992) Zur Prävalenz der Stuhlinkontinenz. Eine vergleichende Untersuchung an Diabetikern und Ulkuskranken. Kontinenz 1: 10-13

22. Ramkumar DP, Rao SSC (2002) Functional anorectal disorders. In: Irvine EJ, Hunt RH (eds) Evidence-based gastroenterology, Decker, Hamilton London, pp 207-222

23. Sahm SW, Lorenz M (2001) Maligne Tumoren der Leber und Gallenwege. In: Caspary WF, Leuschner U, Zeuzem S (Hrsg) Therapie von Leber- und Gallekrankheiten, 2. Aufl. Springer, Berlin Heidelberg New York, S 357-376

24. Schiller LR (1993) Fecal incontinence. In: Sleisenger MH, Fordtran JS (eds) Gastrointestinal diseases, 5 th edn. Saunders, Philadelphia, pp 934-953

25. Schulte-Bockholt A, Schröder O, Koch TR (1999) Obstipation. In: Caspary WF, Stein J (Hrsg) Darmkrankheiten. Springer, Berlin Heidelberg New York, S 141-153

26. Schlieper CA (2000) Grundfragen der Ernährung, 15. Aufl. Handwerk \& Technik, Hamburg

27. Seger W (1995) Chronische Krankheiten des Dickdarms, Morbus Crohn und sozialmedizinische Bedeutung spezieller Symtomkomplexe. In: VDR (Hrsg) Sozialmedizinische Begutachtung in der gesetzlichen Rentenversicherung, 5. Aufl. Gustav Fischer, Stuttgart Jena New York, S 315-318

28. Silverstein FE, Graham DY, Senior JR (1995) Misoprostol reduces serious gastrointestinal complications in patients with rheumatoid arthritis receiving nonsteroidal anti-inflammatory drugs. A randomised, double-blind, placebo-controlled trial. Ann Intern Med 123: 241-249

29. Stein J (1999) Antibiotikaassoziierte Diarrhö und pseudomembranöse Kolitis. In: Caspary WF, Stein J (Hrsg) Darmkrankheiten. Springer, Berlin Heidelberg New York, S 511-518

30. Wight NJD, Hawkey CJ (2001) Nonsteroidal anti-inflammatory drug-related peptic ulcer disease. In: Irvine EJ, Hunt RH (eds) Evidencebased gastroenterology. Decker, Hamilton London, pp 102-118

\section{Vergiftungen durch Schwermetalle und Arsen}

\section{Beitrag von Wegner $\mathrm{R}$ (2001). Der Internist 43:818-827}

\author{
In der Abb. 1 des oben genannten Beitrags ist \\ $\mathrm{Pb} 700$ durch $\mathrm{Pb} 1400$ zu ersetzen.
}

Wir bitten Sie, den Fehler zu entschuldigen. 